\title{
Aprendizaje psicomotriz en el área de Educación Física, Recreación y Deportes mediado por el uso de software educativo \\ Psychomotor learning in the area of Physical Education, Recreation, and Sports, mediated by the use of educational software
}

\author{
Alexandra María Silva Monsalve, Luis Fernando Montañez Sanchéz
}

Universidad de Santander (Colombia)

\begin{abstract}
Resumen. Las escuelas de la era digital son responsables de incluir dentro de sus prácticas cotidianas las Tecnologías de la Información y la Comunicación (TIC); en esa medida, a través de la presente investigación se realiza un proceso de incorporación de TIC al área de Educación Física, Recreación y Deportes para aportar al uso de estas herramientas en este campo académico, brindando solución a la problemática de carencia de uso de TIC en el área deportiva escolar. La herramienta TIC implementada corresponde al software educativo multimedia Ludos. El estudio de tipo cuantitativo correlacional se realizó en Bogotá D.C. con un grupo de 30 estudiantes de grado quinto del Colegio Paraíso Mirador con el objetivo de determinar la eficacia de uso del sobre las habilidades psicomotrices de la población seleccionada. Los resultados se obtuvieron a través de la aplicación de pretest y postest haciendo aplicación de la prueba Piaget-Head y Batería Ozeretsky. Los hallazgos confirmaron que el uso de este software incrementa las habilidades de psicomotricidad, lo cual constituye una oportunidad para demostrar que es posible desarrollar procesos educativos de enseñanza-aprendizaje en el área de Educación Física, incorporando a las clases elementos didácticos que son posibles gracias al desarrollo tecnológico de la era digital.

Palabras clave: coordinación motriz, educación física, lateralidad, psicomotricidad, software educativo.
\end{abstract}

\begin{abstract}
Schools of the digital era are responsible for including Information and Communication Technologies (ICT) within their daily practices. Through the present investigation, a process of incorporation of ICTs in the area of Physical Education, recreation, and sports, is carried out. The main purpose is to use these tools in the academic field, providing solution to the lack of use of ICTs in the school sports field. The Educational Multimedia software «Ludos» was selected for its implementation. This correlational, quantitative study was carried out in the city of Bogotá with a group of 30 fifth-grade students from the «School Paraíso Mirador», with the objective of determining the effectiveness of the mentioned tool in developing psychomotor skills in the selected sample. The results were obtained by a pre-post-test analysis of scores from the Piaget-Head and the Ozeretsky battery tests. The findings confirmed that the use of this software increases psychomotor abilities, which may indicate that it is possible to develop teaching-learning educational processes in the area of physical education by incorporating didactic elements through Technological tools from the digital era.
\end{abstract}

Keywords: educational software, laterality, motor coordination, psychomotricity, physical education.

\section{Introducción}

El desarrollo de la propuesta radica en la motivación de brindar solución a la problemática de la escasa aplicación de las tecnologías de la información y la comunicación (TIC), como apoyo pedagógico en el área de Educación Física. «La problemática en el campo de la Educación Física, surge de la reflexión acerca de qué aspectos específicos relacionados con el conocimiento del contenido debe conocer el profesorado de Educación Física, y también la forma de enseñarlo en las clases « (Almonacid-Fierro, Feu, \& Vizuete, 2018, p. 133). Ahora bien, sobre la forma de enseñar, se ha indagado desde las TIC, en correspondencia a la armonización entre deporte e informática se ha logrado visualizar a través de innumerables elementos, dentro de los que se encuentran los softwares empleados para perfeccionar sistemas de planificación, para el análisis biomecánico de la técnica, para el análisis de acciones de alto nivel de complejidad, para captura de datos por telemetría, y muchos otros de gran valor (Guillen, Herrera, \& De la Rosa, 2018).

No obstante, al hablar de la didáctica de la Educación Física (EF) y la necesidad de incorporar las TIC a las prácticas educativas de enseñanza-aprendizaje donde es limitado su uso, se hace inevitable consolidarla como un apoyo para

Fecha recepción: 13-08-18. Fecha de aceptación: 20-03-19 Alexandra María Silva Monsalve alexandra.silva@cvudes.edu.co la labor docente y una herramienta para la construcción del conocimiento de los estudiantes. De esta manera, «la EF se debe considerar como un conjunto de prácticas físico-deportivas, vinculadas con una preocupación pedagógica» (Molina, Martínez-Baena, \& Villamón, 2017, p.7).

Por otra parte, las dificultades en los procesos de enseñanza de la EF, se han caracterizado por la presencia de clases magistrales con poco apoyo tecnológico. Particularmente en el Colegio Paraíso Mirador, a pesar de encontrarse dotado de infraestructura, se tienen dificultades en cuanto a la proporción de estudiante-equipo. Este problema tiene diversas causas, una de ellas está relacionada con el tema de infraestructura. Así mismo, la inversión a programas de ciencia se ha visto beneficiado por un incremento presupuestal bastante significativo, de acuerdo con lo publicado en la página del Departamento de Planeación (Planeación, 2018). El Gobierno actual ha triplicado la inversión en Ciencia, Tecnología e Innovación; además, también allí se menciona que el 10\% de los fondos del sistema de regalías se va a distribuir para los proyectos de innovación. Sin embargo, la cantidad de estudiantes que se encuentran en colegios distritales o públicos requiere de un esfuerzo mayor por parte del Gobierno para dotar las aulas de herramientas TIC y, sobre todo, iniciar y sostener programas de capacitación docente.

Aunque muchos docentes reporten conocimiento técnico-instrumental en las TIC, la dificultad más notoria radica en su incorporación en las prácticas educativas. Cabero 
(2010) plantea que la formación docente debe encaminarse a cinco áreas: (a) pedagógica; (b) social, ética y legal; (c) técnica; (d) de gestión escolar; (e) de desarrollo profesional. Asimismo, otras de las causas relacionadas con la problemática son: la falta de recursos de calidad para el área de EF, así como la inseguridad de los docentes ante el uso de TIC, debido a que muchas veces los estudiantes tienen mayor dominio de estas herramientas (Cabero, 2004).

Por consiguiente, «el desarrollo de las competencias digitales se ha convertido en todo un problema de investigación que ha despertado gran interés por la comunidad científica a nivel internacional» (Rodríguez-García, Raso, \& RuizPalmero, 2018, p. 75); se menciona que la incorporación de las TIC desarrolla en los estudiantes las competencias de aprender a aprender, además, puede contribuir a desarrollar su capacidad crítica y favorecer su independencia y creatividad (Martínez-Mínguez, 2016)

En el contexto del colegio Paraíso Mirador, además de contar con poca infraestructura, la formación de docentes ha sido muy limitada, por lo cual el uso de las TIC es poco frecuente. Las consecuencias que puede traer la carencia del uso de las TIC en todas las áreas del conocimiento, incluida la educación física, recreación y deportes son: aumento de la brecha digital, entendida esta como diferenciación entre entidades naturales o sociales que acceden a la red y aquellas que no lo hacen, es decir, la desigualdad en uso de TIC, lo cual más adelante puede ocasionar que los estudiantes que no accedieron a TIC en sus procesos educativos sean discriminados a nivel social y laboral (Cabero, 2004). Otras consecuencias son el desconocimiento de los niños acerca de recursos que puedan incrementar su desempeño en actividades físicas, así como una visión de las TIC como herramienta para entretenimiento y ocio en vez de elemento esencial para acceder al conocimiento o a la difusión de información, disminución del trabajo colaborativo, desaprovechamiento de los recursos institucionales existentes y falta de optimización del tiempo en las clases. Para aportar a la solución del problema, en este proyecto se propone el uso de una herramienta NTIC aplicada al área de Educación Física.

La relevancia del proyecto se apoya en la Ley General de Educación, Ley 115 de 1994, donde se plantea que el área de Educación Física es una de las áreas obligatorias y fundamentales para el logro de los objetivos de la educación básica, así como en lo planteado por el Ministerio de Educación Nacional en Colombia en el documento «Orientaciones generales para la educación en tecnología» acerca de visualizar la tecnología con su característica de interdisciplinariedad, ya que así sería de fácil aplicación en todas las áreas consideradas básicas en la educación, razón por la cual esta investigación se considera con alto nivel de pertinencia. El desarrollo de la propuesta se orientó desde la pregunta problematizadora de la investigación: ¿es posible fortalecer el aprendizaje psicomotriz desde el área de Educación Física, Recreación y Deportes en estudiantes implementando el software educativo multimedia interactivo Ludos?

Aunado a la pregunta anterior, surgen dos consideraciones sobre la didáctica de la EF: las TIC deben propiciar el desarrollo de aprendizaje autónomo de estudiantes, pero de igual forma la mejora de las competencias docentes. De esta forma, se ha planteado por algunos autores, «la incorpora- ción de las TIC a la enseñanza y aprendizaje como la acción sistemática y conjunta de diversos recursos didácticos y el apoyo de una organización tutorial, que propician el aprendizaje autónomo de los estudiantes» (Cabero, Llorente, \& Morales-Lozano, 2017, p. 272).

\section{Metodología}

Metodológicamente se adelantó una investigación cuantitativa con un alcance de tipo correlacional, midiendo el comportamiento del grupo en las dos variables a relacionar partiendo de la aplicación de una prueba pretest y postest. El soporte teórico del diseño metodológico está sustentado en la investigación cuantitativa (Hernández, Collado, \& Baptista, 2014), puesto que se realizaron mediciones de las variables en el contexto de la aplicación del recurso educativo interactivo multimedia Ludos, para establecer las conclusiones derivadas de las hipótesis. Corresponde a una investigación correlacional debido a que la investigación pretende conocer la relación entre dos variables en el contexto educativo de la institución Paraíso Mirador IED.

Las variables de esta investigación se estructuran como independiente y dependiente; así, la variable independiente implica la exposición del estudiante a las actividades propuestas en el recurso educativo multimedia Ludos, mientras que la variable dependiente es la habilidad de lateralidad y orientación espacial y desarrollo motor.

La hipótesis a validar en el estudio corresponde a una hipótesis correlacional: «La exposición de los estudiantes ante las actividades del recurso educativo interactivo multimedia Ludos aumenta el nivel de aprendizaje psicomotriz en términos de lateralidad y coordinación dinámica en los estudiantes de quinto grado del Colegio Paraíso Mirador IED»

El recurso implementado - software educativo multimedia interactivo Ludos -, se realizó para este proyecto bajo la mirada de la teoría del aprendizaje constructivista, donde el docente acompaña el proceso y se convierte en un facilitador para la construcción del aprendizaje que el niño realiza a través de su interacción con las actividades propuestas en el recurso. En la teoría constructivista, el niño asume un rol activo y responsable de su aprendizaje bajo la observación y guía del docente (Sánchez-Cortés, García, Sánchez, Moreno, \& Reinoso, 2005); además, la visión del aprendizaje como un proceso activo tiene en cuenta que la manera como se presenta la información cobra importancia en la construcción de conocimiento, esta se plantea con actividades significativas que les permitan aplicar formas para solucionar determinados problemas (Requena, 2008).

El software educativo multimedia interactivo Ludos, es una aplicación interactiva gratuita con un carácter lúdico, que trata con un importante conjunto de contenidos de EF en la Educación primaria. A cada nivel le corresponde un espacio de juego: Diverland (primer ciclo), Ludoland (segundo ciclo) y Moviland (tercer ciclo). Asimismo, el software también incentiva el trabajo colaborativo en los estudiantes, mediante el uso de niveles (Vilamajor \& Esteve, 2016).

Ludos es un recurso educativo elaborado a través del Convenio Internet en el Aula,

entre el Ministerio de Educación y las comunidades au- 
tónomas, por medio del Instituto Nacional de Tecnologías Educativas y de Formación del Profesorado de España (INTEF, 2018). Ludos, se puede constituir como un software en la categoría de los videojuegos, al ser utilizado como material curricular requiere, como otro tipo de materiales, un enfoque pedagógico con unos objetivos y utilidad educativa clara. Tradicionalmente, los materiales (sobre todo libros de texto) se han utilizado como herramientas para suministrar información, pero los videojuegos no cumplen ese objetivo. «Son capaces de trasladar al alumnado a un contexto histórico, deportivo, socio-cultural o geológico simulado para trabajar en la compresión de qué hacer, cómo hacer y por qué hacer una acción con el fin de solucionar un problema» (Gómez, Molina, \& Devís-Devís, 2018, p. 308).

\section{Participantes}

Para la investigación se seleccionó una muestra aleatoria simple, definida como aquella cuya elección de la población se caracteriza porque todos los elementos de esta tienen la misma posibilidad de ser elegidos (Hernández, Collado y Baptista, 2006). Así, en esta investigación es posible seleccionar al azar los estudiantes. Los criterios de inclusión que se tuvieron en cuenta como parámetro para la selección fueron: estudiantes del grado quinto en el colegio Paraíso Mirador IED en la jornada de la mañana y no contar con algún reporte médico o psicológico de déficit atencional o algún tipo de alteración cognitiva que obstaculice los procesos de aprendizaje. Para calcular el tamaño de la muestra se tomó en cuenta lo sugerido Mertens (2005) y Borg y Gall (1989) citados por (Hernández, Collado y Baptista, 2014), quienes plantean que en los estudios cuantitativos de tipo correlacional el tamaño mínimo de la muestra sugerido es de «30 casos por grupo o segmento del universo». En el texto mencionado, se plantea que en los estudios cuantitativos se propone una muestra entre 30 y 50 casos. La población está conformada por estudiantes de ambos géneros: dieciséis de género masculino y catorce de género femenino.

\section{Instrumentos}

Para el pretest y el postest se realizó la aplicación del test de orientación derecha- izquierda BPH, batería Piaget- Head y batería de Ozeretsky. La razón de elección de estos instrumentos deriva de una profunda búsqueda de diferentes instrumentos en psicomotricidad. Fonseca (1988) citado por Baena, Granero y Ruiz (2010) menciona que los instrumentos con los cuales se evalúa el desarrollo motor que los estudiantes y los profesores posean información que puede ser usada para diseñar o cambiar la planeación de los currículos o programas educativos. De esta manera, fueron elegidos los dos instrumentos para la investigación debido a que la prueba de Piaget-Head es aplicable a la edad de la población a investigar y, además, permite valorar habilidades en cuanto a las nociones de derecha- izquierda, en relación con sí mismo y con lo que le rodea. Esta prueba es complementada con la aplicación de la batería de Ozeretsky, la cual es completa en términos motrices debido a que evalúa la coordinación en sus diferentes formas: estática, dinámica de manos, dinámica general, rapidez de movimientos, movimientos simultáneos y ausencia de sincinesias. Además, el rango de edad de aplicación de la batería de Ozeretsky va desde los cuatro hasta los dieciséis años abarca las edades de la población seleccionada.

Test de orientación derecha-izquierda BPH, batería Piaget-Head

El test tiene como objetivo valorar los conocimientos del estudiante en relación con los conceptos de derecha e izquierda sobre sí mismo, los demás y los objetos. Esta prueba está dirigida a niños entre los seis y los doce años de edad y consta de seis pruebas que son aplicadas de forma completa sin tener en cuenta la edad del sujeto. Evalúa psicomotricidad, lateralidad y organización del espacio Baena, Granero y Ruiz (2010). La forma como se califica y valora consiste en considerar correcta la prueba cuando tiene el puntaje esperado en cada prueba. En la primera subprueba se esperan tres puntos de tres posibles; en la segunda se esperan cinco puntos de seis posibles; en la tercera se esperan tres puntos de tres posibles; en la cuarta se esperan seis puntos de ocho posibles; en la quinta se esperan seis puntos de ocho posibles; en la séptima se esperan cinco puntos de seis posibles. En esa medida, el puntaje máximo esperado es 28 y el puntaje máximo posible para obtener es 34.

Batería de Ozeretsky

Es una batería para medir el desarrollo motor dirigida a niños entre los cuatro y los dieciséis años. Su administración es individual y comprende seis subpruebas: coordinación estática, coordinación dinámica de las manos, coordinación dinámica general, rapidez, movimientos simultáneos y precisión en la ejecución. La prueba señala la edad de desarrollo motor del niño. La forma de aplicación de esta prueba se realiza de la siguiente manera: se determina la edad cronológica del niño en meses, luego se aplica la prueba comenzando en la subprueba de la edad inmediatamente anterior, por ejemplo, si el estudiante tiene entre once y doce años se inicia aplicando las subpruebas de diez años, o si tiene diez años se inicia aplicando las subpruebas para nueve años. Si el niño falla en al menos una de las subpruebas para la edad entonces se retrocede una edad y así sucesivamente hasta que no se presenten errores. Una vez el estudiante no presenta errores entonces se continua hasta el final de toda la prueba. Cuando el estudiante no falle en el grupo de subpruebas para la edad entonces es allí donde queda ubicado, determinándose así la edad motora. En el caso de los rangos de edad que dice once-doce años y trececatorce años, para efectos de calificación se toma la edad mayor, es decir, doce años y catorce años.

Los datos se analizaron haciendo uso de software de análisis de datos estadístico. Las matrices de datos tanto del pretest y del postest fueron ingresadas en el software con el fin de determinar estadísticos de tendencia central (moda, media y mediana) y coeficientes de correlación. Una vez determinados los puntajes tanto del pretest y postest como los resultados de las fichas de autoevaluación, se procedió a realizar el análisis de los resultados estableciendo comparaciones entre los resultados previos y posteriores a la intervención con el software. También se realizaron las gráficas de dispersión de datos con el fin de observar la incidencia de la aplicación del programa en el cambio de las variables.

\section{Procedimiento}

El trabajo de investigación aborda diversas fases, así: 
primera fase. Identificación de la problemática a tratar fundamentada básicamente en la nula aplicación de las TIC. Segunda fase. Se presenta un detallado estudio de las bases teóricas que fundamentan el estudio desde la mirada histórica de la inclusión de las TIC a la educación y también el marco investigativo, los antecedentes legales y el marco tecnológico en el que se describen las especificaciones de la herramienta tecnológica que se empleará como soporte pedagógico de la investigación. Tercera fase. Se encuentra el diseño metodológico, las variables, la descripción de la población, el procedimiento y los instrumentos que fueron empleados como evaluación en el pretest y postest, así como la descripción de las técnicas de análisis de datos utilizadas. Cuarta fase. Se presenta el diagnóstico que permitió identificar la situación en cuanto al uso de la tecnología en la institución, así como los datos derivados de la aplicación de los instrumentos seleccionados en la prueba pretest, la cual permitió identificar los niveles de psicomotricidad de los estudiantes. Quinta fase. Se presenta la propuesta pedagógica implementada en las clases de Educación Física que corresponde al software educativo multimedia Ludos, en apartes que muestran el diseño del ambiente de aprendizaje estructurado para potenciar las habilidades que se determinaron a partir del diagnóstico de la población; además del diseño, se muestra la forma en la que la herramienta fue implementada y, como aparte final, se presenta la validación de la propuesta pedagógica que se realizó con la aplicación de los instrumentos determinados previamente para la evaluación postest y los resultados derivados de la autoevaluación de los estudiantes. Sexta fase. Se presenta el análisis de los datos obtenidos de la aplicación de los instrumentos aplicados en la evaluación pretest, la evaluación postest y la ficha de autoevaluación de los estudiantes bajo parámetros estadísticos. Finalmente, se presentan las conclusiones de la investigación, así como las limitaciones presentadas durante el trabajo.

Inicialmente, se realizó una fase de diagnóstico donde se

\begin{tabular}{|c|c|c|c|}
\hline Sesión & $\begin{array}{c}\text { Título de la sección en el } \\
\text { software }\end{array}$ & $\begin{array}{l}\text { Nombres del juego en el } \\
\text { software }\end{array}$ & Temática a tratar \\
\hline s & $\begin{array}{l}\text { Bienvenida y socialización del } \\
\text { software a los estudiantes. } \\
\text { Como mola la video consola }\end{array}$ & $\begin{array}{l}\text { Sesión de bienvenida } \\
\text { ¿Quién encesta más?- } \\
\text { Jugamos al paracaídas. }\end{array}$ & $\begin{array}{l}\text { Normas de convivencia. } \\
\text { Motivación a uso del } \\
\text { software } \\
\text { Coordinación óculo manual. }\end{array}$ \\
\hline 2 & Como mola la video consola & $\begin{array}{l}\text { El copión } \\
\text { Apunta y dispara }\end{array}$ & Coordinación óculo manual \\
\hline 3 & $\begin{array}{l}\text { La gran Pirámide. Ubicado en } \\
\text { la sección del ciclo dos. }\end{array}$ & $\begin{array}{l}\text { Los pozos } \\
\text { Asociar }\end{array}$ & Orientación espacial \\
\hline 4 & $\begin{array}{l}\text { La gran Pirámide } \\
\text { Ubicado en la sección del } \\
\text { ciclo dos. }\end{array}$ & $\begin{array}{l}\text { Laberinto } \\
\text { Orientación } \\
\text { Que no me exploten }\end{array}$ & Orientación espacial \\
\hline I & $\begin{array}{l}\text { La gran Pirámide } \\
\text { Ubicado en la sección del } \\
\text { ciclo dos. }\end{array}$ & $\begin{array}{l}\text { La palabra escondida- } \\
\text { Perseguido }\end{array}$ & Orientación espacial \\
\hline s & $\begin{array}{l}\text { "El templo de los siete } \\
\text { amuletos". Ubicado en la } \\
\text { sección del ciclo dos. }\end{array}$ & $\begin{array}{l}\text { La isla del tesoro- La mina } \\
\text { misteriosa }\end{array}$ & $\begin{array}{l}\text { Desarrollo del pensamiento } \\
\text { aestratégico con capacidades } \\
\text { motrices. }\end{array}$ \\
\hline 7 & $\begin{array}{l}\text { "El templo de los siete } \\
\text { amuletos". Ubicado en la } \\
\text { sección del ciclo dos. }\end{array}$ & $\begin{array}{l}\text { El poder del dominó-El } \\
\text { rayo cósmico. }\end{array}$ & $\begin{array}{l}\text { Desarrollo del pensamiento } \\
\text { estratégico con capacidades } \\
\text { motrices. }\end{array}$ \\
\hline 8 & $\begin{array}{l}\text { "El templo de los siete } \\
\text { amuletos". Ubicado en la } \\
\text { sección del ciclo dos. }\end{array}$ & $\begin{array}{l}\text { La gran escapada - Misión } \\
\text { imposible. }\end{array}$ & $\begin{array}{l}\text { Desarrollo del pensamiento } \\
\text { estratégico con capacidades } \\
\text { motrices. }\end{array}$ \\
\hline s & $\begin{array}{l}\text { "El templo de los siete } \\
\text { amuletos". Ubicado en la } \\
\text { sección del ciclo dos. }\end{array}$ & $\begin{array}{l}\text { Olimpiadas en la Antigua } \\
\text { Grecia. }\end{array}$ & $\begin{array}{l}\text { Desarrollo del pensamiento } \\
\text { estratégico con capacidades } \\
\text { motrices. }\end{array}$ \\
\hline 10 & $\begin{array}{l}\text { "Más alto, fuerte y rápido". } \\
\text { Ubicado en la sección del } \\
\text { ciclo tres. }\end{array}$ & $\begin{array}{l}\text { Flexibilidad-Velocidad - } \\
\text { Resistencia }\end{array}$ & $\begin{array}{l}\text { Conocimiento para } \\
\text { desarrollo y mejora de } \\
\text { cualidades físicas básicas. }\end{array}$ \\
\hline 11 & $\begin{array}{l}\text { "Más alto, fuerte y rápido". } \\
\text { Ubicado en la sección del } \\
\text { ciclo tres. }\end{array}$ & $\begin{array}{l}\text { Fuerza-Completa los } \\
\text { huecos }\end{array}$ & $\begin{array}{l}\text { Conocimiento para } \\
\text { desarrollo y mejora de } \\
\text { cualidades físicas básicas. }\end{array}$ \\
\hline 12 & $\begin{array}{l}\text { "Más alto, fuerte y rápido". } \\
\text { Ubicado en la sección del } \\
\text { ciclo tres. }\end{array}$ & Relaciona - Sopa de letras & $\begin{array}{l}\text { Conocimiento para } \\
\text { desarrollo y mejora de } \\
\text { cualidades físicas básicas. }\end{array}$ \\
\hline
\end{tabular}

aplicó la batería de Ozeretsky y la prueba Piaget-Head. Una vez finalizada la fase de diagnóstico se inicia con la implementación de la estrategia del proyecto Ludos. Se realizó en doce sesiones con duración de 90 minutos cada una, llevadas a cabo una vez por semana durante las clases de Educación Física, Recreación y Deportes en el horario establecido para la población seleccionada en el día de la semana del jueves. Cada una de las sesiones fue dirigida y acompañada por el docente de Educación Física, haciendo uso de la sala de sistemas asignada para la sede de primaria donde estudia la población seleccionada, la cual se encuentra dotada con 40 equipos de cómputo y 40 tabletas. Todos estos equipos tienen software educativo multimedia interactivo Ludos (Tabla 1 y Figura 1).

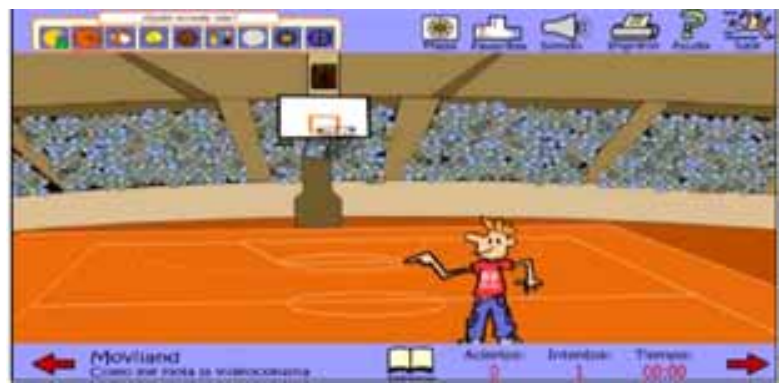

Figura 1. Software educativo Ludos. Fuente: INTEF

Para validar la presente propuesta pedagógica se realizaron dos actividades: La primera consistió en la implementación de 12 sesiones de la propuesta pedagógica con el uso del software educativo multimedia interactivo Ludos. Se procedió a la calificación del instrumento ficha de Autoevaluación, el cual permitía evaluar el nivel de atención y dedicación de los estudiantes, así como la finalización de los niveles de los módulos propuestos en el software. Este instrumento corresponde a la autoevaluación de los estudiantes que se encuentra en el anexo Resultados Ficha de Autoevaluación. El nivel de atención y dedicación fue medido en una escala de 0 a 100 puntos utilizando una escala de Likert donde 1 correspondía a malo, 2 a regular, 3 a bueno y 4 a excelente. Cabe aclarar que en total fueron 12 sesiones con un total de 25 niveles. La segunda actividad consistió en la

\begin{tabular}{|c|c|c|c|c|}
\hline Estudiante & Género & Edad motora en meses & Edad cronológica en meses & Cociente motol \\
\hline 1 & Masculino & 144 & 138 & 104,348 \\
\hline 2 & Femenino & 96 & 128 & 75,000 \\
\hline 3 & Femenino & 108 & 126 & 85,714 \\
\hline 4 & Femenino & 108 & 127 & 85,039 \\
\hline 5 & Masculino & 108 & 140 & 77,143 \\
\hline 6 & Masculino & 108 & 136 & 79,412 \\
\hline 7 & Femenino & 96 & 127 & 75,591 \\
\hline 8 & Femenino & 108 & 155 & 69,677 \\
\hline 9 & Masculino & 96 & 130 & 73,846 \\
\hline 10 & Femenino & 96 & 122 & 78,689 \\
\hline 11 & Masculino & 108 & 138 & 78,261 \\
\hline 12 & Masculino & 108 & 142 & 76,056 \\
\hline 13 & Masculino & 96 & 126 & 76,190 \\
\hline 14 & Masculino & 108 & 142 & 76,056 \\
\hline 15 & Femenino & 96 & 126 & 76,190 \\
\hline 16 & Masculino & 96 & 128 & 75,000 \\
\hline 17 & Femenino & 96 & 123 & 78,049 \\
\hline 18 & Femenino & 96 & 136 & 70,588 \\
\hline 19 & Femenino & 96 & 126 & 76,190 \\
\hline 20 & Femenino & 84 & 114 & 73,684 \\
\hline 21 & Femenino & 96 & 124 & 77,419 \\
\hline 22 & Masculino & 96 & 142 & 67,606 \\
\hline 23 & Femenino & 108 & 132 & 81,818 \\
\hline 24 & Femenino & 108 & 154 & 70,130 \\
\hline 25 & Masculino & 108 & 135 & 80,000 \\
\hline 26 & Masculino & 108 & 142 & 76,056 \\
\hline 27 & Masculino & 108 & 154 & 70,130 \\
\hline 28 & Masculino & 96 & 140 & 68,571 \\
\hline 29 & Masculino & 96 & 129 & 74,419 \\
\hline 30 & Masculino & 96 & 126 & 76,190 \\
\hline
\end{tabular}


aplicación de los instrumentos Batería Ozeretsky y Batería Piaget-Head como post-test.

\section{Análisis estadístico de los datos}

Para el diagnóstico de la población se realizó la aplicación de una encuesta de percepción a los docentes y de un pretest y un postest a los estudiantes. Se utilizaron los test de orientación derecha-izquierda BPH y la batería de Ozeretsky (Tabla 2).

Sobre los resultados del pretest, se muestra desarrollo bajo en cuatro estudiantes, quienes mostraron dificultades significativas en la ejecución de la prueba. La Tabla 1 muestra las puntuaciones directas obtenidas en la aplicación de la prueba de Ozeretsky y en la Tabla 2 se puede observar la gráfica que muestra los resultados del cociente motor arrojados por la prueba de Ozeretsky y que brindan la interpretación de estos datos.

Tabla 2 .

Resultados prueba de Ozeretsky pre-test a través de estadísticos de tendencia central.

\begin{tabular}{lc}
\multicolumn{1}{c}{ Estadísticos de tendencia Central } & Resultados Prueba Ozeretsky en pretest \\
\hline Promedio & 76,769 \\
\hline Mediana & 76,123 \\
\hline Moda & 76,1904762 \\
\hline Fuente: Autor del proyecto
\end{tabular}

Asimismo, se evidenciaron puntajes que muestran desarrollo básico en diecisiete estudiantes, lo cual determina que estos estudiantes poseen las habilidades básicas de lateralidad y orientación espacial de acuerdo con la edad. Las puntuaciones más bajas muestran que se presenta un desarrollo bajo en cuatro estudiantes, pues mostraron dificultades significativas en la ejecución de la prueba. La Tabla 3 muestra los resultados directos obtenidos de la aplicación de la prueba Piaget-Head en el pretest.

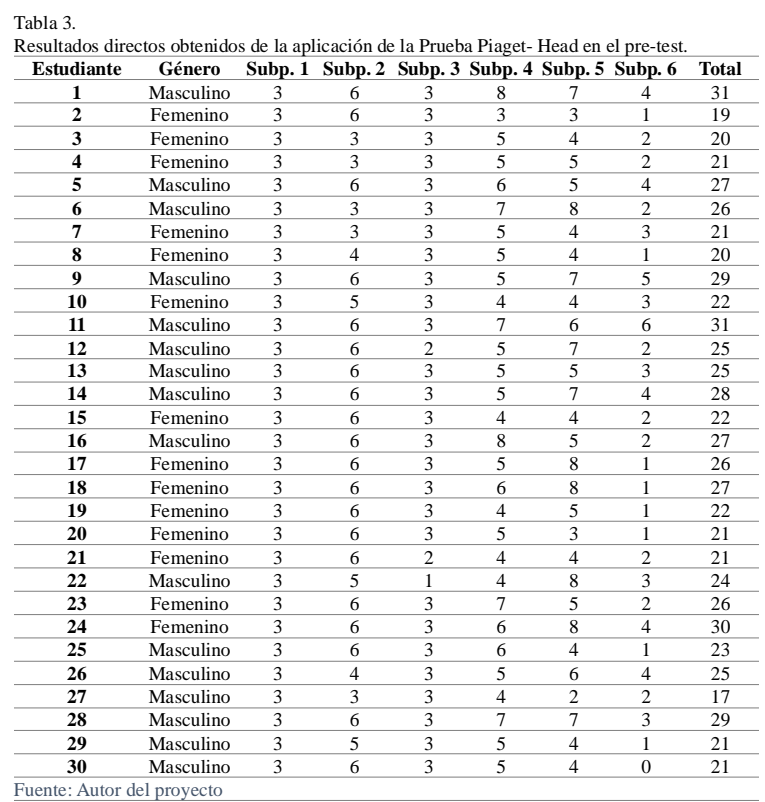

La aplicación de las pruebas Ozeretsky y Piaget Head dieron como resultado que la población de género masculino obtiene un puntaje mínimamente superior en cuanto a las habilidades de lateralidad, orientación espacial y coordinación general.

\section{Análisis de resultados}

La comparación de los resultados muestra que durante el pretest de la prueba Piaget Head, cuatro estudiantes obtuvieron puntajes inferiores a 70 puntos, mientras que en el postest todos los estudiantes obtuvieron resultados superiores. De igual forma, los resultados indican que entre $70 \%$ y $80 \%$, se encontraban los resultados de dieciséis estudiantes en el pretest y en el postest únicamente un estudiante obtuvo este puntaje, el resto de los estudiantes se encontraban con un resultado entre 80 y 100.

Los resultados estadísticos de tendencia central, muestran un aumento en el promedio de los resultados por cada una de las pruebas, indicando que hay una mejora en las puntuaciones mejorando en 17.16 puntos. La mediana indica que los datos se distribuyen uniformemente, es decir, que con la aplicación del software hubo incremento en las habilidades de todos los estudiantes. Con respecto a la moda, los resultados indican que en ambos casos (pretest y postest) la moda se mantiene cerca del promedio y se observa mejoría con la aplicación del software.

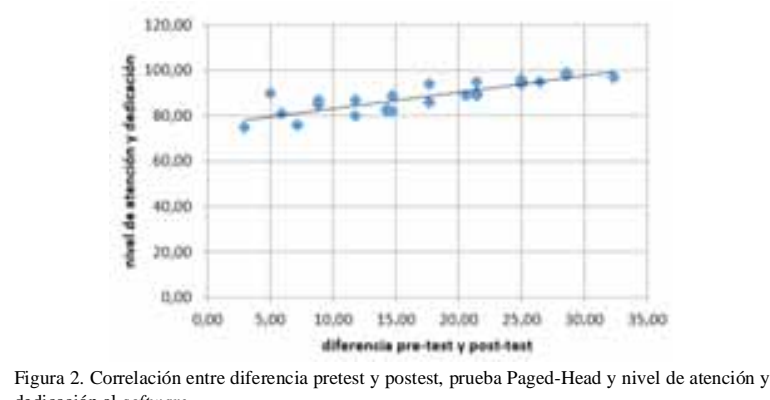
Figura 2. Correlación e
dedicación al software

La Figura 2 muestra que el nivel de atención se correlaciona de manera positiva con los incrementos en la ejecución de la prueba del Piaget-Head. La correlación fue de .85, lo cual significa que los niños que se autoevaluaron con excelente y buen desempeño obtuvieron en el postest de la prueba Piaget-Head buenos resultados. De igual forma, el factor de finalización de los niveles en el software se correlaciona de manera positiva con los incrementos en la ejecución de la prueba del Piaget-Head. La correlación fue de .78, lo cual indica que los niños que finalizaron más niveles obtuvieron en el postest de la prueba Piaget-Head mejores resultados que aquellos que no terminaron los niveles.

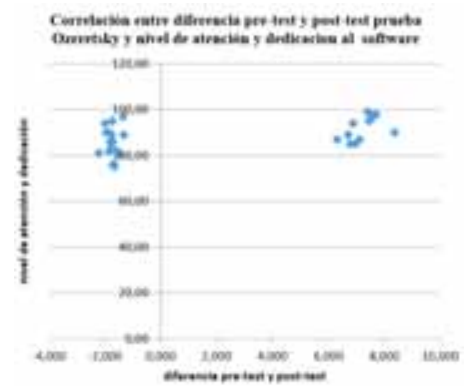

Figura 3. Correlación prueba Ozertsky y niveles de atención y dedicación del software

La Figura 3 muestra la correlación entre la diferencia de pretest y postest en la batería de Ozeretsky con el nivel de atención y dedicación al software. Los resultados indican que el nivel de correlación en muy bajo, .45, significa que la percepción de atención y diligencia en las actividades del software no determinó el incremento en las habilidades de coordinación medidas en la batería. También se estableció la correlación entre la diferencia de pretest y postest en la bate- 
ría de Ozeretsky con la cantidad de niveles terminados en el software. Los resultados indican que el nivel de correlación es muy bajo, .42, significa que la finalización de las actividades o niveles del software no determina el incremento en las habilidades de coordinación medidas en la batería.

\section{Discusión}

Se evidenció al incorporar las TIC en la didáctica de la EF, la mejora en el aprendizaje psicomotriz, especialmente: ubicación derecha - izquierda; más que en procesos de coordinación. Es importante resaltar sobre los anteriores resultados, el papel que deben cumplir tanto docentes y estudiantes, en procesos didácticos, para este caso en la enseñanza de la EF. Se enfatiza el papel de mediador, que el maestro debe adoptar en el proceso de enseñanza-aprendizaje. Por otra parte, la motivación es el motor que impulsa al alumno a reflexionar, investigar o crear sus propias propuestas. Sin embargo, no es fácil romper la inercia y pensar que el profesor no enseña, son los alumnos que construyen su propio aprendizaje (Pérez-López, Rivera, \& Trigueros, 2017).

Los resultados de la aplicación de la ficha de autoevaluación respaldan lo encontrado en los estudios realizados por Gordejo (2004), San Martín (2004), Campos, Garrido y Castañeda (2009) que muestran que las TIC, específicamente el uso del software, es adecuado para incrementar la motivación de los estudiantes, lo cual se refleja en el alto nivel de asistencia a las sesiones propuestas con el uso del software Ludos y el alto número de niveles finalizados por cada uno de los estudiantes. Estos estudios mencionados también son respaldados por el incremento en el aprendizaje psicomotriz de los estudiantes, especialmente en las habilidades de lateralidad y orientación espacial, el cual se observó de manera clara con la comparación entre los resultados del pretest y postest de la prueba Piaget-Head. El diligenciamiento de la ficha de autoevaluación por parte de los estudiantes, así como la interpretación de sus resultados en un ámbito global, muestra que el estudiante tuvo un papel activo en su propio proceso de aprendizaje, que constituye también una idea de la teoría constructivista.

Los resultados también apoyan lo concluido por Añel (2009), quien en su investigación incluyó el software EduSport en la facultad de Ciencias de la Educación y Deporte, lo que produjo un incremento en el nivel académico. Este apoyo al estudio está determinado por el incremento del nivel de habilidades psicomotrices que constituyen aprendizaje psicomotriz. Al igual, lo apoyan resultados obtenidos en la investigación sobre la importancia de usar software en el juego de entrenamiento de voleibol (Adin-Mariana \& Cojocaru, 2015).

Los hallazgos de la presente investigación tienen una alta relación con los planteamientos de la teoría del constructivismo en cuanto a la significación de las tareas realizadas. A pesar de que las tareas se llevaron a cabo en el computador y las pruebas fueron con otros materiales, la transferencia del conocimiento fue exitosa. El permitir a los estudiantes llevar a cabo los procesos de autoevaluación y autorreflexión en torno a su propio desempeño ante el software y sus procesos de atención y dedicación validan lo planteado en los principios de la teoría del aprendizaje autorregulado, en relación con el control de la voluntad y el establecimiento de objetivos para cumplir con la tarea. Finalmente, los resultados indican la consecución de aprendizajes significativos en lo que respecta a las habilidades de lateralidad más que a las habilidades de coordinación general medidas por la batería de Ozeretsky.

\section{Conclusiones}

La era digital ha transformado las dinámicas de aprendizaje de los niños que se desarrollan en estos tiempos. Las TIC a pesar de estar muchas veces en las escuelas no son aprovechadas al máximo, especialmente en cuanto a los currículos del área de educación física, recreación y deportes. Los estudiantes en la búsqueda de herramientas TIC para las diversas áreas del aprendizaje e incluso los momentos de tiempo libre invierten muchas horas frente a los equipos de cómputo, lo cual impide o dificulta el desarrollo de las habilidades físicas.

Esta investigación permitió brindar solución a la carencia de uso de TIC en el área de EF a través de la implementación del software educativo multimedia Ludos, de la cual se pudo concluir que la investigación dio cumplimiento al objetivo general planteado, ya que el software Ludos fortaleció el aprendizaje psicomotriz de los estudiantes de grado quinto que participaron en el estudio.

Los instrumentos de medida seleccionados dan cuenta de una medida objetiva de las habilidades que se buscaban potencializar con la implementación del software, además el tipo de metodología Pretest-Postest permitió evidenciar los cambios en las estructuras de pensamiento de los estudiantes en cuanto a la psicomotricidad, lo cual valida el primer objetivo específico de la investigación. El software Ludos muestra mejores resultados en pruebas de habilidad de orientación espacial y lateralidad como la prueba Piaget-Head en comparación con los resultados en pruebas que miden las habilidades de coordinación como la batería de Ozeretsky, lo cual permite el cumplimiento del segundo objetivo específico de la investigación en cuanto a la eficacia y pertinencia de la herramienta tecnológica seleccionada. Es posible desarrollar algunos conceptos teóricos y prácticos relacionados con la psicomotricidad a través de la incorporación de una herramienta TIC como el software.

La investigación permitió abordar las habilidades psicomotrices en los diferentes niveles que se plantearon a lo largo del marco teórico, haciendo referencia a la teoría de Lázaro y Berruezo sobre la pirámide del desarrollo humano (Lázaro \& Berruezo, 2009). En la base de la pirámide de la psicomotricidad se encuentran los fundamentos laberintico vestibulares que aportan a la orientación básica y la propiocepción con los movimientos articulares requeridos para el manejo del mouse. Asimismo, en este primer nivel los resultados muestran que el software aporta en la vinculación de estados emocionales adecuados a las temáticas tratadas, en el segundo nivel de la pirámide de psicomotricidad se encuentra la lateralidad cuya mejora fue evidente con el uso del software; en cuanto el tercer nivel de la psicomotricidad, el uso del software Ludos permite que los estudiantes desarrollen coordinación visomotriz, de forma que exista una coordinación entre lo visto en pantalla y las órdenes que deben 
ser dadas con el mouse o las flechas del teclado.

Finalmente, en cuanto al último nivel de procesos superiores, el software permitió desarrollar procesos de atención, cuyos resultados fueron positivos en los estudiantes y especialmente la autonomía, ya que una vez iniciada la sesión los niños ingresaban al módulo del software y de acuerdo con el tiempo establecido cada uno pasaba los niveles siguiendo su propio ritmo de aprendizaje. Además, muchos de los estudiantes reportaban al docente de educación física el ingreso al software desde sus casas lo que permite desarrollar adecuados niveles de autonomía. Esta conclusión valida el tercer objetivo específico de la investigación ya que los resultados permiten determinar la eficacia y pertinencia del software Ludos como apoyo para mejorar los niveles de psicomotricidad.

Frente a la problemática de la carencia de uso de TIC en el área de EF, la investigación cumplió el objetivo general en tanto permitió determinar a través de la experiencia de implementación del software Ludos que es posible y pertinente hacer uso de las TIC como apoyo a los procesos de enseñanza-aprendizaje beneficiando a los estudiantes en la mejora y potenciación de sus habilidades psicomotrices. Las niñas mostraron un índice ligeramente mayor que los niños en el incremento de las habilidades entre el pretest y postest con las pruebas aplicadas. Esto probablemente se debe a un mayor nivel de atención y disciplina en la ejecución de cada una de las tareas propuestas en el software.

Los niños mostraron un alto nivel de interés y motivación en el uso de la herramienta software en la clase de Educación Física, lo cual se notó en los resultados y mediciones que se realizaron con el instrumento de autoevaluación y en un nivel de asistencia del $100 \%$ en las doce sesiones de aplicación del software Ludos. La posibilidad de retroalimentación del software permite que cada estudiante obtenga una retroalimentación de su desempeño de manera inmediata y constante, la cual se da a través de los sonidos e indicaciones del software ante la presencia de alguna equivocación o desempeño incorrecto por parte de los estudiantes, lo cual fortalece el aprendizaje.

Los resultados demuestran que la implementación de nuevas tecnologías para optimizar el aprendizaje de habilidades psicomotrices en los estudiantes debe ser una prioridad en las escuelas y colegios, debido a que dichas herramientas permiten que los estudiantes sean artífices de la construcción de su propio conocimiento en un ambiente de novedad que redunda en aprendizaje significativo. Por tanto, esta investigación, se puede recomendar a docentes en las áreas de EF para orientar los entrenamientos buscando una mejora en la capacidad decisional del deportista en las diferentes acciones de juego (Conejero, Claver, Fernández-Echeverría, Gil-Arias, \& Moreno, 2017).

\section{Trabajos futuros}

La investigación presentada permite que el área de EF a porte en la construcción de conocimiento práctico y teórico en relación con el uso de las herramientas tecnológicas que surgen en la era digital. Por esto se recomienda que para futuras investigaciones en el tema se involucren poblaciones de menor edad donde el desarrollo psicomotriz inicia de manera que sean más evidentes los avances logrados con la implementación de las TIC. Durante la investigación se observa que el uso continuado del mouse y de las flechas de dirección del teclado puede ser un factor que fortalece las habilidades en orientación espacial y lateralidad. Sin embargo, esta variable no fue analizada y se sugiere sea tenida en cuenta en estudios posteriores que pretendan potenciar esta habilidad en estudiantes de básica primaria. Además, es importante la inclusión de instrumentos de medida cuyos resultados no dependan directamente de la edad cronológica de los estudiantes, ya que con la aplicación de la Batería de Ozeretsky los pequeños avances no son evidentes en los resultados del post-test.

Algunos reportes verbales de los estudiantes indicaban que ellos accedían a la herramienta desde sus casas a través del Internet. De esta manera, la utilización de las TIC permite convertir el aula tradicional en el aula virtual, y permite motivar a los estudiantes durante todo el desarrollo del proceso e involucrados en su trabajo al propio ritmo de aprendizaje, haciendo accesible el conocimiento a cada estudiante (Silva, Mendoza \& Girado, 2018, p.124). Lo cual puede constituirse en una variable a medir en las futuras investigaciones. La consulta bibliográfica en el marco investigativo evidenció una amplia gama de herramientas TIC aplicables al área de educación física, razón por la cual los docentes de esta área pueden incorporar herramientas TIC en el desarrollo de sus clases y aportar en el proceso investigativo al difundir los resultados de sus experiencias.

Esta investigación motiva a los docentes del área a implementar las TIC para el desarrollo de habilidades físicas alejándose de las ideas de generación de sedentarismo en los estudiantes y aprovechando los recursos que han sido construidos para apoyar la educación. «En el campo pedagógico queda mucho trabajo por explorar para formar en el autoaprendizaje y en el trabajo colaborativo» (Martínez \& Silva, 2018, p.99).Por otra parte, para complementar la didáctica en la enseñanza de la EF, se propone la relación de los estilos de aprendizaje (Pacheco \& Maldonado, 2017) y la utilización de las TIC, «se pueden implementar análisis psicométrico de los estilos de enseñanza, así como los estilos participativos, individualizadores, creativos y socializadores « (Merino-Barrero, Valero-Valenzuela, \& Moreno-Murcia, 2017, p. 227).

\section{Referencias}

Adin-Mariana, C., \& Cojocaru, M. (2015). The importance use of resources software in the game of volleyball training. World, 6th International Conference EDUWORLD 2014 - Education Facing Contemporary, 12351241.

Almonacid-Fierro, A., Feu, S., \& Vizuete, M. (2018). Validación de un cuestionario para medir el Conocimiento Didáctico del Contenido en el profesorado de Educación Física. Retos. Nuevas tendencias en Educación Física, Recreación y Deportes, 132-137.

Añel, M. (2009). La utilidad de los recursos tecnológicos institucionales en las clases de educación física. Revista de Forma

Baena, A., Granero, A. y Ruiz, P. (2010). Procedimientos e 
instrumentos para la medición y evaluación del desarrollo motor en el sistema educativo. Associated Editors, 2(2), 63-76.

Cabero, J. (2004). Reflexiones sobre la brecha digital y la educación. En F. Soto y J. Rodríguez (Coord.), Tecnología, educación y diversidad: retos y realidades de la inclusión social, (pp. 23-42). Murcia: Consejería de Educación y Cultura.

Cabero, J. (2014). Formación del profesorado universitario en TIC. aplicación del método delphi para la selección de los contenidos formativos. Educación XX1, 111-132.

Cabero, J., Llorente, C., \& Morales-Lozano, J. (2017). Evaluación del desempeño docente en la formación virtual: ideas para la configuración de un modelo. Revista Iberoamericana de Educación a Distancia. RIED, 260-279.

Cabero-Almenara, J. (2010). Los retos de la integración de las TICs en los procesos educativos: Límites y posibilidades. Perspectiva educacional, 49(1), 32-61.

Campos, M. y Garrido, M. y Castañeda, C. (2009). Técnicas para promover el aprendizaje significativo en Educación Física: los Cmap-Tools. Retos: nuevas tendencias en educación física, deporte y recreación, (16), 58-62.

Conejero, M., Claver, F., Fernández-Echeverría, C., Gil-Arias, *., \& Moreno, P. (2017). Toma de decisiones y rendimiento en las acciones de juego intermedias y finalistas en voleibol, en sets con diferente resultado. Retos. Nuevas Tendencias en Educación Física, Deportes y Recreación, 28-33.

Congreso de la República de Colombia. (1994). Ley General de Educación. [Ley 115 de 1994]. Recuperado de http:// www.mineducacion.gov.co/1759/articles124745_archivo_pdf9.pdf

Gómez, f., Molina, P., \& Devís-Devís, J. (2018). Los videojuegos como materiales curriculares: una aproximación a su uso en Educación Física. Retos. Nuevas tendencias en Educación Física, Recreación y Deportes, 305-310.

Gordejo, F. (2004). El ordenador como recurso de enseñanzaaprendizaje en Educación Física. Lecturas: Educación física y deportes, (77), 17.

Guillen, L., Herrera, A., \& De la Rosa, Y. (2018). Las herramientas tecnológicas TIC's como elemento alternativa para el desarrollo del componente físico. Retos. Nuevas tendencias en Educación Física, Recreación y Deportes, 222-229.

Hernández, R., Collado, C., \& Baptista, P. (2014). Metodología de la investigación. 6a Edición. México: Mc Graw Hill.

INTEF. (2018). Internet en el aula. Obtenido de http:// recursostic.educacion.es/primaria/ludos/web/

Lázaro, A., \& Berruezo, P. (2009). La pirámide del desarrollo humano. Revista Iberoamericana de Psicomotricidad y Técnicas Corporales, 15-42.

Martínez-Mínguez, L. (2016). Proyectos de Aprendizaje Tutorados y autoevaluación de competencias profesionales en la formación inicial del profesorado. Retos. Nuevas tendencias en Educación Física, Deporte y Recreación, 242-250.

Martínez, E., Silva, A. (2018). Los MOOC: una revisión de la literatura para orientar su gestión. Revista Perspectiva Histórica. 7 ,12(19,) 99-124. Recuperado de http:// www.perspectivahistorica.com.br/revistas/ 1542328438.pdf

Merino-Barrero, J., Valero-Valenzuela, A., \& Moreno-Murcia, J. (2017). Análisis psicométrico del cuestionario estilos de enseñanza en educación física (EEEF) . Revista Internacional de Medicina y Ciencias de la Actividad Física y el Deporte, 225-241.

Molina, P., Martínez-Baena, A., \& Villamón, M. (2017). Pedagogía de la Educación Física: análisis de las revistas españolas más productivas (2005-2014)R. Retos. Nuevas tendencias en Educación Física, Deportes y Recreación, 14-18.

Pacheco, R., \& Maldonado, E. (2017). Relación entre los estilos de aprendizaje y las teorías de enseñanza. Retos. Nuevas Tendencias en Educación Física Recreación y Deportes, 7-13.

Pérez-López, J., Rivera, E., \& Trigueros, C. (2017). «The prophecy of the chosen ones»: an example of gamification applied to university teaching. Revista Internacional de Medicina y Ciencias de la Actividad Física y el Deporte, 243-260.

Planeación, D. N. (2018). DNP. Obtenido de https:// www.dnp.gov.co/

Requena, S. (2008). El modelo constructivista con las nuevas tecnologías, aplicado en el proceso de aprendizaje. RUSC. Universities and Knowledge Society Journal, 5(2), 6.

Rodríguez-García, A., Raso, F., \& Ruiz-Palmero, J. (2018). Competencia digital, educación superior y formación del profesorado: un estudio de meta-análisis en la Web of Science. PIXEL BIT. Revista de Medios y Educación, 6581.

San Martin, P. (2014). Software educativo para mejorar el proceso de enseñanza aprendizaje de la metodología de los deportes individuales: gimnasia, natación y atletismo dirigido a los estudiantes de la carrera de cultura física de la Universidad Nacional.

Sánchez-Cortés, García, A., Sánchez, J., Moreno, P., \& Reinoso, A. (2005). Research Developments in Learning Technologies . Obtenido de https:// www.researchgate.net/publication/237245882

Silva, A., Mendoza, G, \& Girado, A (2018). Prevención del consumo de sustancias psicoactivas. Un aporte desde la neurociencia y el aprendizaje basado en proyectos ABP. Revista Iberoamericana De Educación, 78(1), 107126. Obtenido de https://rieoei.org/RIE/article/view/3214

Vilamajor, M., \& Esteve, F. (2016). Dispositivos móviles y aprendizaje cooperativo: diseño de una intervención con dispositivos móviles en un entorno de aprendizaje cooperativo en la etapa de educación primaria. EDUTEC, 1135-9250.

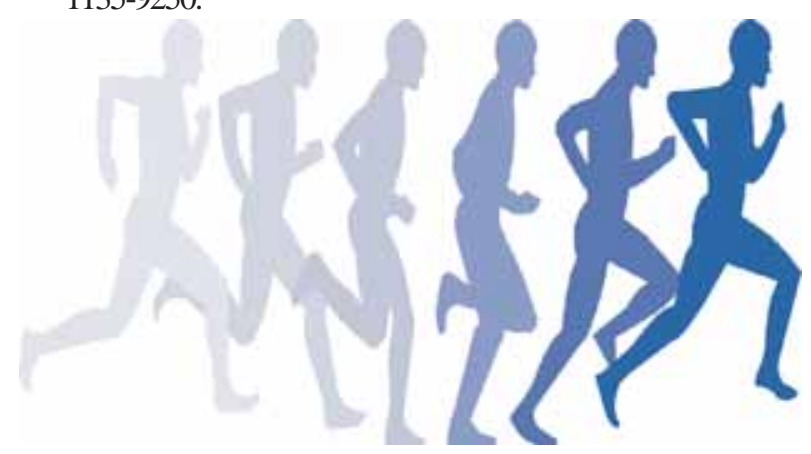

